\title{
Multiple States in Turbulent Large-Aspect-Ratio Thermal Convection: What Determines the Number of Convection Rolls?
}

\author{
Qi Wang $\odot,{ }^{1,2}$ Roberto Verzicco, ${ }^{3,4,1}$ Detlef Lohse ${ }^{1,5, *}$ and Olga Shishkina $\odot^{5, \dagger}$ \\ ${ }^{1}$ Physics of Fluids Group and Max Planck Center for Complex Fluid Dynamics, MESA+ Institute \\ and J. M. Burgers Centre for Fluid Dynamics, University of Twente, P.O. Box 217, 7500AE Enschede, Netherlands \\ ${ }^{2}$ Department of Modern Mechanics, University of Science and Technology of China, Hefei 230027, China \\ ${ }^{3}$ Dipartimento di Ingegneria Industriale, University of Rome "Tor Vergata", Via del Politecnico 1, 00133 Roma, Italy \\ ${ }^{4}$ Gran Sasso Science Institute-Viale F. Crispi, 767100 L'Aquila, Italy \\ ${ }^{5}$ Max Planck Institute for Dynamics and Self-Organization, 37077 Göttingen, Germany
}

(Received 3 April 2020; accepted 20 July 2020; published 12 August 2020)

\begin{abstract}
Wall-bounded turbulent flows can take different statistically stationary turbulent states, with different transport properties, even for the very same values of the control parameters. What state the system takes depends on the initial conditions. Here we analyze the multiple states in large-aspect ratio $(\Gamma)$ twodimensional turbulent Rayleigh-Bénard flow with no-slip plates and horizontally periodic boundary conditions as model system. We determine the number $n$ of convection rolls, their mean aspect ratios $\Gamma_{r}=\Gamma / n$, and the corresponding transport properties of the flow (i.e., the Nusselt number $\mathrm{Nu}$ ), as function of the control parameters Rayleigh (Ra) and Prandtl number. The effective scaling exponent $\beta$ in $\mathrm{Nu} \sim \mathrm{Ra}^{\beta}$ is found to depend on the realized state and thus $\Gamma_{r}$, with a larger value for the smaller $\Gamma_{r}$. By making use of a generalized Friedrichs inequality, we show that the elliptical shape of the rolls and viscous damping determine the $\Gamma_{r}$ window for the realizable turbulent states. The theoretical results are in excellent agreement with our numerical finding $2 / 3 \leq \Gamma_{r} \leq 4 / 3$, where the lower threshold is approached for the larger Ra. Finally, we show that the theoretical approach to frame $\Gamma_{r}$ also works for free-slip boundary conditions.
\end{abstract}

DOI: 10.1103/PhysRevLett.125.074501

For laminar flows, flow transitions can often be calculated from linear stability analysis. Such an analysis not only gives the critical value of the control parameter at which the instability sets in, but it also gives the wavelength of the emerging structure. Famous classical examples for linearly unstable wall-bounded flows are the onset of convection rolls in Rayleigh-Bénard convection or the onset of Taylor rolls in Taylor-Couette flow [1]. In both cases, the rolls of the most unstable mode have a certain wavelength which follows from the linear stability analysis. With increasing flow driving strength, more and more modes become unstable, and in the fully turbulent case the base flow is unstable to basically any perturbation.

What then sets the size of the flow structures in such fully turbulent wall-bounded flow? Several findings have suggested that wall-bounded turbulent flows can take different statistically stationary turbulent states, with different length scale of the flow structures and with different transport properties, even for the very same values of the control parameters. Examples for the coexistence of such multiple turbulent states include turbulent (rotating) Rayleigh-Bénard convection [2-8], Taylor-Couette turbulence [9-11], double-diffusive convection turbulence [12], von Karman flow [13-16], rotating spherical Couette flow [17], Couette flow with span-wise rotation [18], but also geophysical flows $[19,20]$ such as in ocean circulation [21-23], in the liquid metal core of Earth [24-27], or in the atmosphere $[28,29]$.

The occurrence of multiple states in fully turbulent flows can be considered unexpected since, according to Kolmogorov [30], for strong enough turbulence, the fluctuations should become so strong that the whole highly dimensional phase space is explored. Of course, one could argue that in the above given cases and examples, the turbulence driving has not yet been strong enough to reach that state and that the occurrence of multiple turbulent states in wall-bounded turbulence may be a finite size effect, but in any case even then it remains open what sets the range of allowed sizes of the flow structures in such turbulent flows.

To illuminate this question, as a model system we picked two-dimensional (2D) Rayleigh-Bénard (RB) turbulence, the flow in a closed container heated from below and cooled from above [31-33]. The control parameters are the Rayleigh number Ra, which is the dimensionless temperature difference between the plates, the Prandtl number Pr, which is the ratio between kinematic viscosity $(\nu)$ and thermal diffusivity $(\kappa)$, and the aspect ratio $\Gamma=W / H$, i.e., the ratio between horizontal $(W)$ and vertical $(H)$ extension of the system. The response parameters are the Nusselt 
number $\mathrm{Nu}=Q H /(k \Delta)$ and the Reynolds number $\operatorname{Re}=U H / \nu$, which indicate the dimensionless heat transport and flow strength in the system. Here $Q$ is the heat flux crossing the system, $k$ the thermal conductivity, $\Delta$ the temperature difference at the plates, and $U=\sqrt{\left\langle\mathbf{u}^{2}\right\rangle_{V, t}}$ the time and volume-averaged velocity.

The flow dynamics is given by the Boussinesq approximation of the Navier-Stokes and the advection-diffusion equation, with the corresponding boundary conditions (BCs) for the temperature and velocity fields. For the latter at the plates we will first apply no-slip BCs, but later also examine free-slip BCs-a difference which will turn out to be major for the range of allowed states. Periodic BCs are used in the horizontal direction.

We are very much aware of the differences between 2D and 3D RB flow [34], but in particular for large $\operatorname{Pr} \geq 1$ there are extremely close similarities between $2 \mathrm{D}$ and $3 \mathrm{D}$ RB flows, and we wanted to pick a model system for which (i) we can reasonably explore the considerable parameter space for a large enough number of initial flow conditions and (ii) we have the chance to obtain exact analytical results for the range of allowed flow structures.

The parameter range we will explore is for large Prandtl numbers in the range $1 \leq \operatorname{Pr} \leq 100$, for Rayleigh numbers in the range $10^{7} \leq \mathrm{Ra} \leq 10^{10}$ and for large $\Gamma$ up to $\Gamma=32$. Note that in 2D RB, multiple coexisting turbulent states had been found before for $\operatorname{Ra}=10^{7}, \operatorname{Pr}=0.7$ and $\Gamma \approx 0.64$ (i.e., in an extremely limited range of the parameter space) [3], but not for such large $\Gamma$ and $\mathrm{Ra}$ as we explore here, as the range of chosen initial flow conditions was not large enough [4], and clearly not as general and omnipresent as we will find here.

The direct numerical simulations were done with an advanced finite difference code [35] with the criteria for the grid resolution, as found to be required in Ref. [36]. The code has extensively been tested and benchmarked against other codes [37] and applied in 2D RB even up to very large $\mathrm{Ra}=4.64 \times 10^{14}[38,39]$. More simulation details for all explored cases can be found in the Supplemental Material. In order to trigger the possible convection roll state, we use different initial roll states generated by a Fourier basis: $u(x, z)=\sin \left(n^{(i)} \pi x / \Gamma\right) \cos (\pi z), w(x, z)=-\cos \left(n^{(i)} \pi x / \Gamma\right)$ $\sin (\pi z)$, where $n^{(i)}$ indicates the initial number of rolls in the horizontal direction. The initial temperature has a linear profile with random perturbations.

In Figs. 1(a) and 1(b) we show the temporal evolution of some flow characteristics for six different initial flow conditions for the case of $\mathrm{Ra}=10^{10}, \operatorname{Pr}=10$, and $\Gamma=8$. We vary the initial number $n^{(i)}$ of rolls from $n^{(i)}=$ 4 to $n^{(i)}=14$, implying aspect ratios of the initial rolls from $\Gamma_{r}^{(i)}=\Gamma / n^{(i)}=2$ to $\Gamma_{r}^{(i)}=4 / 7$. As flow characteristics we picked the Reynolds number ratio $\mathrm{Re}_{z} / \mathrm{Re}_{x}$ and the Nusselt number $\mathrm{Nu}$. Here $\operatorname{Re}_{z}=\sqrt{\left\langle w^{2}\right\rangle_{V}} H / \nu$ is the

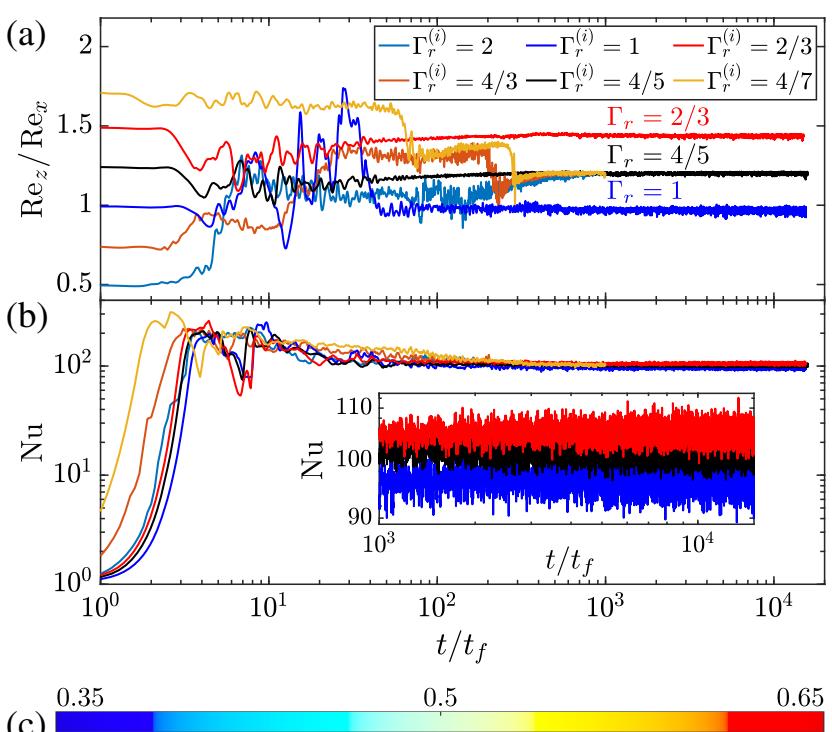

(c)

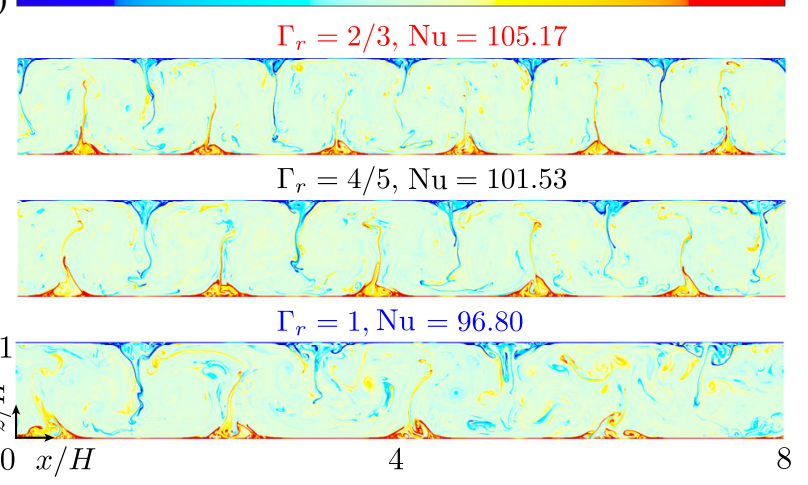

FIG. 1. (a), (b) Time evolution of (a) $\mathrm{Re}_{z} / \mathrm{Re}_{x}$ and (b) $\mathrm{Nu}$ for different initial roll states, $\operatorname{Ra}=10^{10}, \operatorname{Pr}=10, \Gamma=8 . \Gamma_{r}^{(i)}=$ $\Gamma / n^{(i)}$ is the initial and $\Gamma_{r}=\Gamma / n$ the final mean aspect ratio of the rolls; $n^{(i)}$ is the initial and $n$ the final number of the rolls. Note the logarithmic scales of the time axes. (c) Snapshots of the temperature fields for the three statistically stable turbulent states for $\Gamma_{r}=1(n=8), \Gamma_{r}=4 / 5(n=10)$, and $\Gamma_{r}=2 / 3(n=12)$ and the corresponding Nusselt numbers, for $\mathrm{Ra}=10^{10}, \operatorname{Pr}=10$, and $\Gamma=8$. All subfigures are for no-slip BCs.

volume averaged vertical Reynolds number and $\mathrm{Re}_{x}=$ $\sqrt{\left\langle u^{2}\right\rangle_{V}} H / \nu$ the horizontal one, where $w(t)$ and $u(t)$ are the respective velocities. As one can see in Figs. 1(a) and 1(b), depending on the six initial conditions, over the very long time of more than $10^{4}$ free fall time units, the system evolves to either of three different final turbulent states, all with different Reynolds number ratio $\mathrm{Re}_{z} / \mathrm{Re}_{x}$ and Nusselt number $\mathrm{Nu}$. The smaller the final mean aspect ratio $\Gamma_{r}$ of the rolls, the larger the Reynolds number ratio $\mathrm{Re}_{z} / \mathrm{Re}_{x}$ and $\mathrm{Nu}$, due to more plume-ejecting regions which have strong vertical motion.

The time evolution of some of the six different initial states $\left(n^{(i)}=6,14\right)$, analyzed in Figs. 1(a) and 1(b), can be seen in the Supplemental Movies [40], displaying roll splitting and merging events. The states with large initial rolls (corresponding to $n^{(i)}=4,6$ ) break up quickly, while 

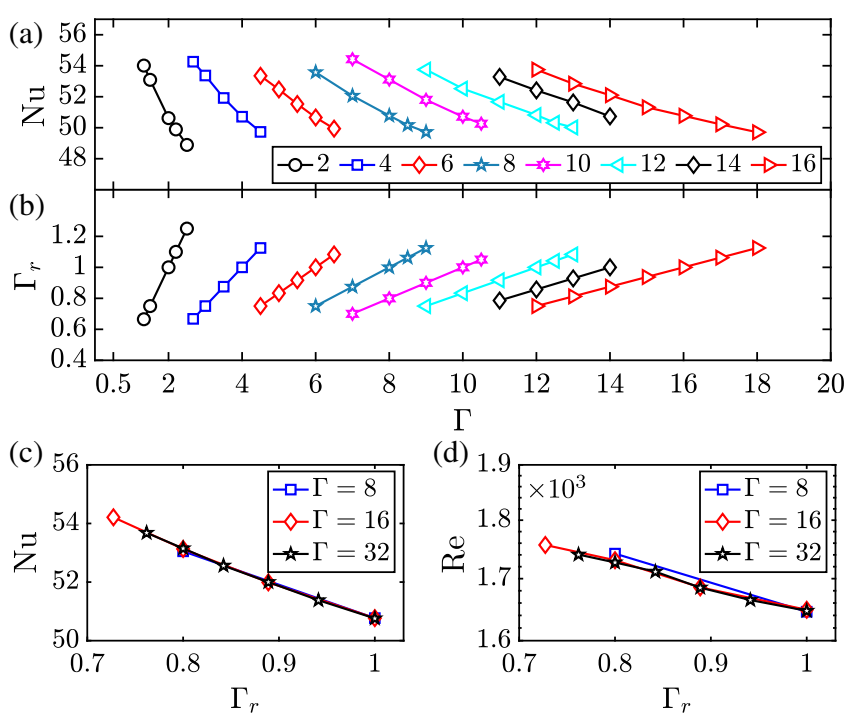

FIG. 2. (a) $\mathrm{Nu}$ and (b) final aspect ratio $\Gamma_{r}=\Gamma / n$ of individual rolls, as function of $\Gamma$, for different turbulent states. The numbers and colors in the legend denote the number $n$ of convection rolls of that state. (c) $\mathrm{Nu}$ and (d) Re as functions of $\Gamma_{r}=\Gamma / n$ for three different values of $\Gamma$. In this figure $\operatorname{Ra}=10^{9}$ and $\operatorname{Pr}=10$ and the BCs are no slip.

those with small initial rolls $\left(n^{(i)}=14\right)$ first undergo a transition into an unstable 12-roll state (with smaller $\mathrm{Re}_{z} / \mathrm{Re}_{x}$ than the stable one) as seen in Fig. 1(a), followed by merging events of two neighboring convection rolls. Though both the Reynolds number ratio and the Nusselt number keep on fluctuating in time, reflecting the turbulent nature of the states, the three final statistically stable turbulent states are clearly distinguished. We characterize them by the final aspect ratio of their rolls, namely $\Gamma_{r}=1, \Gamma_{r}=4 / 5$, and $\Gamma_{r}=2 / 3$, corresponding to $n=\Gamma / \Gamma_{r}=8,10$, and 12 rolls, respectively. Snapshots of these states and their corresponding Nusselt numbers are shown in Fig. 1(c). As one can see, the larger the number of rolls, the better the (heat) transport properties of the system, a characteristics which was found in Taylor-Couette flow before [9] and which can intuitively be understood, due to the larger number of emitted plumes at the interfaces between the rolls.

Consequently, when the cell aspect ratio $\Gamma$ is stretched, the stretching of the mean aspect ratio $\Gamma_{r}=\Gamma / n$ of the corresponding individual rolls is accompanied with a decrease of the corresponding Nusselt number, as seen in Figs. 2(a) and 2(b). Though this behavior has been seen before [4], in Figs. 2(a) and 2(b), we clearly see the coexistence of the different turbulent states. The determining relevance of the final mean aspect ratio $\Gamma_{r}=\Gamma / n$ of the individual rolls for the Nusselt number $\mathrm{Nu}$ and Reynolds number Re in the statistically stationary case is nicely demonstrated in Figs. 2(c) and 2(d), where we show that the dependences $\mathrm{Nu}\left(\Gamma_{r}\right)$ and $\operatorname{Re}\left(\Gamma_{r}\right)$ are universal and irrespective of the individual values of $\Gamma$ or $n$.
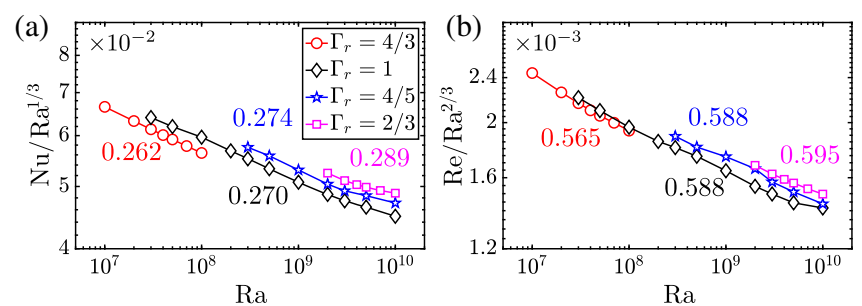

FIG. 3. (a) Compensated Nusselt number $\mathrm{Nu} / \mathrm{Ra}^{1 / 3}$ and (b) compensated Reynolds number $\mathrm{Re} / \mathrm{Ra}^{2 / 3}$, as functions of $\mathrm{Ra}$, for four different turbulent states as characterized by $\Gamma_{r}$, for $\operatorname{Pr}=10, \Gamma=8$, and no-slip BCs. The effective scaling exponents $\beta$ and $\gamma$ in the scaling relations $\mathrm{Nu} \sim \mathrm{Ra}^{\beta}$ and $\mathrm{Re} \sim \mathrm{Ra}^{\gamma}$ are shown next to the curves in the respective color of the state and curve.

Remarkably, not only the absolute value of the Nusselt number depends on $\Gamma_{r}$, but even the effective scaling behavior of $\mathrm{Nu}$ with $\mathrm{Ra}$, as can be seen in Fig. 3(a). The same holds for the Reynolds number, Fig. 3(b). In both cases the effective scaling exponent is larger for turbulent states with smaller mean aspect ratio $\Gamma_{r}$ of the rolls [see the values given in Figs. 3(a) and 3(b)]; i.e., when the system can accommodate a larger number $n=\Gamma / \Gamma_{r}$ of rolls, presumably reflecting the larger disorder and the larger number of emitted plumes for those states.

From Figs. 3(a) and 3(b), we also see that turbulent states with a too large aspect ratio $\Gamma_{r}$ of their rolls cease to exist with increasing Ra. Which turbulent states-as characterized by the mean aspect ratio $\Gamma_{r}$ of their rolls-are statistically stable for given $\mathrm{Ra}$ and $\mathrm{Pr}$ can be seen from the phase diagrams in Fig. 4. For fixed $\operatorname{Pr}=10$, all statistically stable turbulent states in the no-slip case have
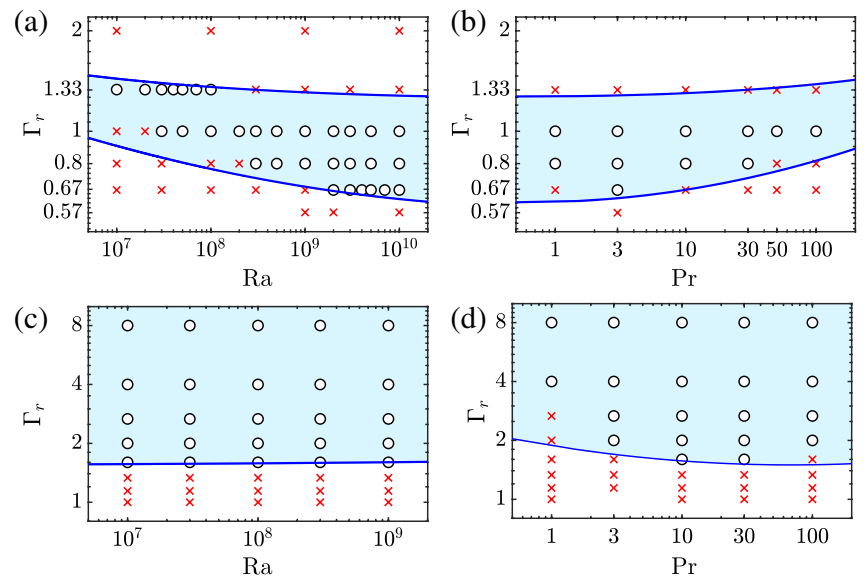

FIG. 4. Phase diagram in the (a),(c) $\mathrm{Ra}-\Gamma_{r}$ and (b),(d) $\operatorname{Pr}-\Gamma_{r}$ parameter space for (a),(b) no-slip BCs and (c),(d) free-slip BCs: (a) $\operatorname{Pr}=10, \Gamma=8$; (b) $\mathrm{Ra}=10^{9}, \Gamma=8$; (c) $\operatorname{Pr}=10, \Gamma=16$; (d) $\mathrm{Ra}=10^{8}, \Gamma=16$. Black circles denote that the corresponding roll state is stable, whereas red crosses mean that it is not stable. The theoretical estimates for the transitions between the regimes are shown as solid lines. 
an aspect ratio $\Gamma_{r}$ in the range $2 / 3 \leq \Gamma_{r} \leq 4 / 3$, in the Rarange analyzed in this Letter. With increasing $\mathrm{Ra}$, we see the range moving towards smaller values of $\Gamma_{r}$, e.g., with $1 \leq \Gamma_{r} \leq 4 / 3$ for $\mathrm{Ra}=10^{8}$ and $2 / 3 \leq \Gamma_{r} \leq 1$ for $\mathrm{Ra}=10^{10}$, see Fig. 4(a). For Ra $=10^{9}$, we find $2 / 3 \leq \Gamma_{r} \leq 1$ for all $\mathrm{Pr}$ analyzed in this Letter, see Fig. 4(b).

We now set out to mathematically understand the range of $\Gamma_{r}$ the system can take for given control parameters. First, we recall that the roll size in 3D flows [41] is restricted by the elliptical instability [42-44]. Similarly, the strain-vorticity balance in 2D flows is determined by the elliptical shape of the rolls. Thus we assume that the essence of the flow is a set of elliptical rolls, each of which can be described by a stream function $\Psi(x, z)=$ $(\xi+\eta)\left(z^{2} / 2\right)+(\xi-\eta)\left(x^{2} / 2\right)$ with $\xi \geq \eta \geq 0$.

The aspect ratio of the rolls, $\Gamma_{r}$, is directly related to the strain $\eta$ and (half of) the vorticity $\xi$ through the relation $\Gamma_{r}=\sqrt{(\xi+\eta) /(\xi-\eta)}$, corresponding to $\eta / \xi=$ $\left(\Gamma_{r}^{2}-1\right) /\left(\Gamma_{r}^{2}+1\right)$. Averaging $u^{2}$ and $w^{2}$ over the area $\left[-\Gamma_{r} H / 2, \Gamma_{r} H / 2\right] \times[-H / 2, H / 2]$, where $u(x, z)=\partial \Psi /$ $\partial z$ and $w(x, z)=-\partial \Psi / \partial x$, we obtain $\operatorname{Re}_{x}=\Gamma_{r} \operatorname{Re}_{z}$, which is in agreement with the simulations, see [40].

The Reynolds number of the roll, $\mathrm{Re}_{r}$, satisfies

$$
\operatorname{Re}^{2} \geq \operatorname{Re}_{r}^{2}=\operatorname{Re}_{x}^{2}+\operatorname{Re}_{z}^{2}=\xi(\xi+\eta) H^{4} /\left(6 \nu^{2}\right) .
$$

To form a roll, the strain must be bounded by the vorticity, $\eta \leq \xi$, which gives $\operatorname{Re}^{2} \geq \eta^{2} H^{4} /\left(3 \nu^{2}\right)$. We now make use of the exact global balance for the total enstrophy $\Omega^{2}$ and the mean kinetic energy dissipation rate $\epsilon_{u}$ [45], namely, $4 \nu \xi^{2}=\nu \Omega^{2}=\epsilon_{u}=\nu^{3} H^{-4}(\mathrm{Nu}-1) \mathrm{Ra} \mathrm{Pr}^{-2}$. With the definition

$$
B \equiv \operatorname{Re}^{2} \operatorname{Pr}^{2} \operatorname{Ra}^{-1}(\mathrm{Nu}-1)^{-1}
$$

(which can be seen as nondimensionalized ratio between the kinetic energy and the energy dissipation rate), from the relation between $\eta / \xi$ and $\Gamma_{r}$ and the inequalities (1) and $\eta \leq \xi$ we obtain $B \geq\left(\Gamma_{r}^{2}-1\right)^{2} /\left(\Gamma_{r}^{2}+1\right)^{2} / 12$, which gives an estimate of the maximal size of the rolls as

$$
\Gamma_{r} \leq \sqrt{1+2 \sqrt{3 B}} / \sqrt{1-2 \sqrt{3 B}} .
$$

Figures 5(a) and 5(b) show the Ra and Pr dependences of $B$, obtained numerically for the no-slip BCs, as well as their data fits (see [40]). As seen from the phase diagrams in Figs. 4(a) and 4(b), using these fits in the upper bound (3) gives quite reasonable estimates for the maximal mean roll size of statistically stable turbulent states, see upper lines in Figs. 4(a) and 4(b).

We now come to the lower bound of the window of allowed $\Gamma_{r}$. First note that $\Gamma_{r}$ cannot be infinitesimally small, because, in order to form the rolls, the following
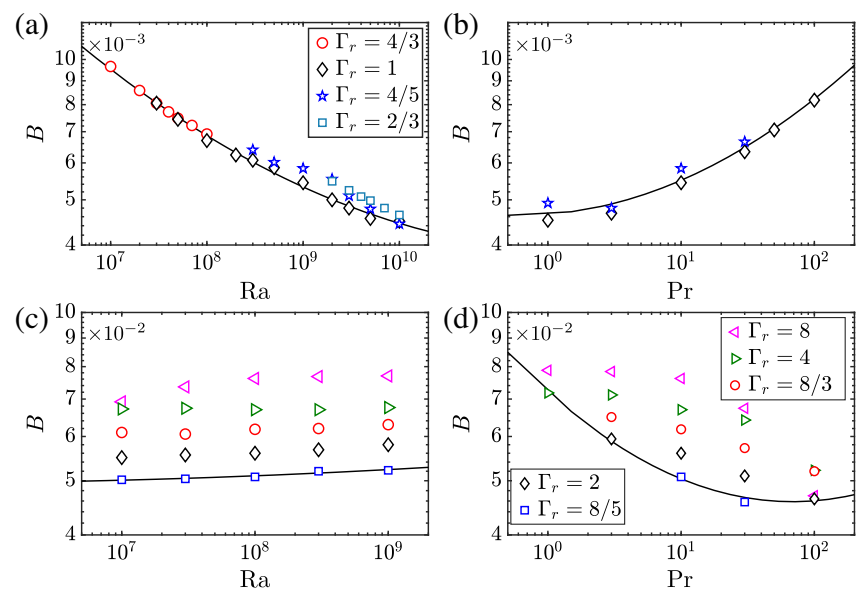

FIG. 5. $\quad B=\left\{\left[\operatorname{Re}^{2} \operatorname{Pr}^{2}\right] /[\operatorname{Ra}(\mathrm{Nu}-1)]\right\}$, as functions of (a),(c) $\mathrm{Ra}$ and (b),(d) Pr, for (a),(b) no-slip BCs and (c),(d) free-slip BCs. (a) $\operatorname{Pr}=10, \Gamma=8$; (b) $\operatorname{Ra}=10^{9}, \Gamma=8$; (c) $\operatorname{Pr}=10, \Gamma=16$; (d) $\mathrm{Ra}=10^{8}, \Gamma=16$. The solid lines are fits to the data (see the Supplemental Material [40]). Note that the values of $B$ in the noslip case (a),(b) are much smaller than those in the free-slip case (c), (d).

should hold: $\Gamma_{r} \geq \sqrt{1-2 \sqrt{3 B}} / \sqrt{1+2 \sqrt{3 B}}$. One can obtain a more accurate estimate when considering a rectangular region that frames a particular roll. Under the assumption that the velocity components achieve their maximum at the boundaries of this rectangle or vanish there, there exists a certain constant $c>2$ such that $c^{2} \overline{\mathbf{u}^{2}} \leq \Gamma_{r}^{2} H^{2} \overline{(\nabla \mathbf{u})^{2}}$. We call this relation the generalized Friedrichs inequality and derive it in the Supplemental Material [40]. This inequality gives as estimate for the lower bound of $\Gamma_{r}$,

$$
\Gamma_{r} \geq c \sqrt{B}
$$

which is plotted as lower lines in Figs. 4(a) and 4(b) for $c=9$. As can be seen, the theoretical slopes reflect the general tendency of the numerical results. Note however that at this point we cannot calculate the absolute value of the transition, i.e., the value of $c$. We also remark that in the case when the container is too slender to keep two rolls [with the size, according to (4)]; i.e., when $\Gamma<2 c \sqrt{B}$, the flow can take the form of a zonal flow only. Thus, in the noslip case, the zonal flow configuration [46,47] is possible, but only in small-aspect ratio containers.

Our approach also leads to reasonable estimates for the window of allowed statistically stable states in the free-slip case, which was numerically analyzed in Ref. [48]: taking the dependences $\mathrm{Nu}(\mathrm{Ra}, \mathrm{Pr})$ and $\mathrm{Re}(\mathrm{Ra}, \mathrm{Pr})$ for the various roll states from that work, with the definition (2) we obtain $B(\mathrm{Ra}, \mathrm{Pr})$, see Figs. 5(c) and 5(d). In this case, the values of $B$ are significantly larger, allowing much larger rolls than in the no-slip case. Furthermore, when the inequality (1) holds 
for any $\eta$, also when the strain is as strong as the vorticity, $\eta=\xi$, the rolls can be of any size $1 \leq \Gamma_{r} \leq \Gamma / 2$ and the zonal flows are possible as well. This situation happens when $B \geq 1 / 12$. Thus, very large- $\Gamma_{r}$ states are possible, while particular state realizations depend on the initial conditions. Indeed, this is consistent with the numerical findings in Ref. [48], which are shown in Figs. 4(c) and 4(d). Note in particular that in Ref. [48] an as large stable state as $\Gamma_{r}=64$ (for $\Gamma=128, \quad \operatorname{Ra}=10^{8}, \quad \operatorname{Pr}=10$ ) was found.

What about the lower bound for allowed $\Gamma_{r}$ in the freeslip case? In contrast to the upper bound, it always exists and just as in the no-slip case, by arguments again based on the generalized Friedrich inequality (see the Supplemental Material [40]), we can find it. In Figs. 4(c) and 4(d) the result for the smallest $\Gamma_{r}$ is plotted. It is based on the estimate (4) and uses the fits from Figs. 5(c) and 5(d) for the smallest values of $B$ with $c=7$. Again, the theoretical slopes in the $\Gamma_{r}-\mathrm{Ra}$ and $\Gamma_{r}-\operatorname{Pr}$ phase diagrams reflect the general trend of the numerical results.

In conclusion, we have numerically shown the coexistence of multiple statistically stable states in turbulent RB convection with no-slip BCs, with different mean aspect ratios of their turbulent rolls and different transport properties, even scaling wise. We then theoretically illuminated what principles determine the allowed window of the mean size of the turbulent convection rolls (and thus their absolute number), occurring from the ellipticity of the convection rolls. These criteria also work for the free-slip case.

Even though a 2D model may seem somehow artificial, there are various cases in which the flow dynamics is mostly 2D, e.g., because of geometrical confinement, stratification, or background rotation. Therefore our model in itself is relevant, but the main ideas of our approach can also be generalized to other wall-bounded turbulent flows, such as rotating Rayleigh-Bénard flow, Taylor-Couette flow, Couette flow with span-wise rotation, double diffusive convection, etc., and also to geophysical flows such as those mentioned in the introduction. They may also give guidance for turbulence flow control, in order to predict which turbulent states are feasible to be realizable.

K. L. Chong, L. Liu, R. Stevens, A. Barker, and A. Tilgner are gratefully acknowledged for discussions. We also acknowledge the Twente Max-Planck Center, the Deutsche Forschungsgemeinschaft (Priority Programme SPP 1881 “Turbulent Superstructures"), PRACE for awarding us access to MareNostrum based in Spain at the Barcelona Supercomputing Centre (BSC) under PRACE Project No. 2018194742. This work was partly carried out on the national e-infrastructure of SURFsara, a subsidiary of SURF cooperation, the collaborative ICT organization for Dutch education and research. Q. W. acknowledges financial support from China Scholarship Council (CSC) and National Natural Science Foundation of China (Grant No. 11621202). *d.lohse@utwente.nl

†lga.Shishkina@ds.mpg.de

[1] P. Drazin and W.H. Reid, Hydrodynamic Stability (Cambridge University Press, Cambridge, England, 1981).

[2] H.-D. Xi and K.-Q. Xia, Flow mode transitions in turbulent thermal convection, Phys. Fluids 20, 055104 (2008).

[3] E. P. van der Poel, R. J. A. M. Stevens, and D. Lohse, Connecting flow structures and heat flux in turbulent Rayleigh-Bénard convection, Phys. Rev. E 84, 045303(R) (2011).

[4] E. P. van der Poel, R. J. A. M. Stevens, and D. Lohse, Flow states in two-dimensional Rayleigh-Bénard convection as a function of aspect-ratio and Rayleigh number, Phys. Fluids 24, 085104 (2012).

[5] S. Weiss and G. Ahlers, Effect of tilting on turbulent convection: cylindrical samples with aspect ratio $\Gamma=0.50$, J. Fluid Mech. 715, 314 (2013).

[6] Q. Wang, Z.-H. Wan, R. Yan, and D.-J. Sun, Multiple states and heat transfer in two-dimensional tilted convection with large aspect ratios, Phys. Rev. Fluids 3, 113503 (2018).

[7] Y.-C. Xie, G.-Y. Ding, and K.-Q. Xia, Flow Topology Transition via Global Bifurcation in Thermally Driven Turbulence, Phys. Rev. Lett. 120, 214501 (2018).

[8] B. Favier, C. Guervilly, and E. Knobloch, Subcritical turbulent condensate in rapidly rotating Rayleigh-Bénard convection, J. Fluid Mech. 864, R1 (2019).

[9] S. G. Huisman, R. C. A. van der Veen, C. Sun, and D. Lohse, Multiple states in highly turbulent Taylor-Couette flow, Nat. Commun. 5, 3820 (2014).

[10] R. C. van der Veen, S. G. Huisman, O.-Y. Dung, H. L. Tang, C. Sun, D. Lohse, Exploring the phase space of multiple states in highly turbulent Taylor-Couette flow, Phys. Rev. Fluids 1, 024401 (2016).

[11] R. Ostilla-Mónico, D. Lohse, and R. Verzicco, Effect of roll number on the statistics of turbulent Taylor-Couette flow, Phys. Rev. Fluids 1, 054402 (2016).

[12] Y. Yang, W. Chen, R. Verzicco, and D. Lohse, Multiple states and transport properties of double-diffusive convection turbulence, Proc. Natl. Acad. Sci. U.S.A. 117, 14676 (2020).

[13] F. Ravelet, L. Marié, A. Chiffaudel, and F. Daviaud, Multistability and Memory Effect in a Highly Turbulent Flow: Experimental Evidence for a Global Bifurcation, Phys. Rev. Lett. 93, 164501 (2004).

[14] F. Ravelet, M. Berhanu, R. Monchaux, S. Aumaitre, A. Chiffaudel, F. Daviaud, B. Dubrulle, M. Bourgoin, P. Odier, N. Plihon, J. F. Pinton, R. Volk, S. Fauve, N. Mordant, and F. Petrelis, Chaotic Dynamos Generated by a Turbulent Flow of Liquid Sodium, Phys. Rev. Lett. 101, 074502 (2008).

[15] P. Cortet, A. Chiffaudel, F. Daviaud, and B. Dubrulle, Experimental Evidence of a Phase Transition in a Closed Turbulent Flow, Phys. Rev. Lett. 105, 214501 (2010).

[16] D. Faranda, Y. Sato, B. Saint-Michel, C. Wiertel, V. Padilla, B. Dubrulle, and F. Daviaud, Stochastic Chaos in a Turbulent Swirling Flow, Phys. Rev. Lett. 119, 014502 (2017).

[17] D. S. Zimmerman, A. A. Triana, and D. P. Lathrop, Bi-stability in turbulent, rotating spherical Couette flow, Phys. Fluids 23, 065104 (2011). 
[18] Z. Xia, Y. Shi, Q. Cai, M. Wan, and S. Chen, Multiple states in turbulent plane Couette flow with spanwise rotation, J. Fluid Mech. 837, 477 (2018).

[19] F. Bouchet and E. Simonnet, Random Changes of Flow Topology in Two-Dimensional and Geophysical Turbulence, Phys. Rev. Lett. 102, 094504 (2009).

[20] F. Bouchet and A. Venaille, Statistical mechanics of twodimensional and geophysical flows, Phys. Rep. 515, 227 (2012).

[21] W. S. Broecker, D. M. Peteet, and D. Rind, Does the oceanatmosphere system have more than one stable mode of operation?, Nature (London) 315, 21 (1985).

[22] M. J. Schmeits and H. A. Dijkstra, Bimodal behavior of the Kuroshio and the Gulf stream, J. Phys. Oceanogr. 31, 3435 (2001).

[23] A. Ganopolski and S. Rahmstorf, Abrupt Glacial Climate Changes due to Stochastic Resonance, Phys. Rev. Lett. 88, 038501 (2002).

[24] G. A. Glatzmaiers and P. H. Roberts, A three-dimensional self-consistent computer simulation of a geomagnetic field reversal, Nature (London) 377, 203 (1995).

[25] J. Li, T. Sato, and A. Kageyama, Repeated and sudden reversals of the dipole field generated by a spherical dynamo action, Science 295, 1887 (2002).

[26] P. L. Olson, R. S. Coe, P. E. Driscoll, G. A. Glatzmaier, and P.H. Roberts, Geodynamo reversal frequency and heterogeneous core-mantle boundary heat flow, Phys. Earth Planet. Interiors 180, 66 (2010).

[27] A. Sheyko, C. C. Finlay, and A. Jackson, Magnetic reversals from planetary dynamo waves, Nature (London) 539, 551 (2016).

[28] E. R. Weeks, Y. Tian, J. Urbach, K. Ide, H. L. Swinney, and M. Ghil, Transitions between blocked and zonal flows in a rotating annulus with topography, Science 278, 1598 (1997).

[29] F. Bouchet, J. Rolland, and E. Simonnet, Rare Event Algorithm Links Transitions in Turbulent Flows with Activated Nucleations, Phys. Rev. Lett. 122, 074502 (2019).

[30] A. N. Kolmogorov, On degeneration (decay) of isotropic turbulence in incompressible viscous liquid, Dokl. Akad. Nauk SSSR 31, 538 (1941).

[31] G. Ahlers, S. Grossmann, and D. Lohse, Heat transfer and large scale dynamics in turbulent Rayleigh-Bénard convection, Rev. Mod. Phys. 81, 503 (2009).

[32] D. Lohse and K.-Q. Xia, Small-scale properties of turbulent Rayleigh-Bénard convection, Annu. Rev. Fluid Mech. 42, 335 (2010).

[33] F. Chilla and J. Schumacher, New perspectives in turbulent Rayleigh-Bénard convection, Eur. Phys. J. E 35, 58 (2012).

[34] E. P. van der Poel, R. J. A. M. Stevens, and D. Lohse, Comparison between two- and three-dimensional RayleighBénard convection, J. Fluid Mech. 736, 177 (2013).
[35] E. P. van der Poel, R. Ostilla-Mónico, J. Donners, and R. Verzicco, A pencil distributed finite difference code for strongly turbulent wall-bounded flows, Comput. Fluids 116, 10 (2015).

[36] O. Shishkina, R. J. A. M. Stevens, S. Grossmann, and D. Lohse, Boundary layer structure in turbulent thermal convection and its consequences for the required numerical resolution, New J. Phys. 12, 075022 (2010).

[37] G. L. Kooij, M. A. Botchev, E. M. Frederix, B. J. Geurts, S. Horn, D. Lohse, E. P. van der Poel, O. Shishkina, R. J. A. M. Stevens, and R. Verzicco, Comparison of computational codes for direct numerical simulations of turbulent Rayleigh-Bénard convection, Comput. Fluids 166, 1 (2018).

[38] X. Zhu, V. Mathai, R. J. A. M. Stevens, R. Verzicco, and D. Lohse, Transition to the Ultimate Regime in TwoDimensional Rayleigh-Bénard Convection, Phys. Rev. Lett. 120, 144503 (2018).

[39] X. Zhu, V. Mathai, R. J. A. M. Stevens, R. Verzicco, and D. Lohse, Reply to Absence of Evidence for the Ultimate Regime in Two-Dimensional Rayleigh-Bénard Convection, Phys. Rev. Lett. 123, 259402 (2019).

[40] See the Supplemental Material at http://link.aps.org/ supplemental/10.1103/PhysRevLett.125.074501 for movies, numerical details, derivation of the generalized Friedrichs inequality, effective scaling exponents, and fits of functions $B$.

[41] L. Zwirner, A. Tilgner, and O. Shishkina, Elliptical Instability and Multi-Roll Flow Modes of the LargeScale Circulation in Confined Turbulent Rayleigh-Bénard Convection, Phys. Rev. Lett. 125, 054502 (2020).

[42] M. Landman and P. Saffman, The three-dimensional instability of strained vortices in a viscous fluid, Phys. Fluids 30, 2339 (1987).

[43] F. Waleffe, On the three-dimensional instability of strained vortices, Phys. Fluids A 2, 76 (1990).

[44] R. R. Kerswell, Elliptical instability, Annu. Rev. Fluid Mech. 34, 83 (2002).

[45] B. I. Shraiman and E. D. Siggia, Heat transport in high-Rayleigh number convection, Phys. Rev. A 42, 3650 (1990).

[46] E. P. van der Poel, R. Ostilla-Mónico, R. Verzicco, and D. Lohse, Effect of velocity boundary conditions on the heat transfer and flow topology in two-dimensional Rayleigh-Bénard convection, Phys. Rev. E 90, 013017 (2014).

[47] D. Goluskin, H. Johnston, G. R. Flierl, and E. A. Spiegel, Convectively driven shear and decreased heat flux, J. Fluid Mech. 759, 360 (2014).

[48] Q. Wang, K.-L. Chong, R. J. A. M. Stevens, R. Verzicco, and D. Lohse, From zonal flow to convection rolls in Rayleigh-Bénard convection with free-slip plates, arXiv: 2005.02084 . 